\title{
TECHNOLOGY BASED OVERVIEW ON SOFTWARE TESTING TRENDS, TECHNIQUES, AND CHALLENGES
}

\author{
Aishwarya Sundaram, \\ University of Malaya, \\ Malaysia
}

\begin{abstract}
Software testing is a process of evaluating the developed software against the actual requirements from the customer. Software development methodology has transformed based on numerous driving factors over decades and so is the software testing methodologies.

Software remains inevitable in rapid growth of technologies in various fields like academy, medicine, media, safety, agriculture, robotics, gaming, aviation, national defence etc., In this paper, the existing and emerging trends, techniques, and issues of software testing are discussed based on the technologies.
\end{abstract}

Keywords: Latest technologies, testing techniques, automation testing, challenges in testing, and software testing life cycle.

\section{INTRODUCTION}

Software testing is the process of evaluating the system with the specifications provided by the customer. It is a quality assurance process of finding bugs in the developed software that aid developers to deliver the code error-free. Fig1. Represents the phases of software testing life cycle. Each of the phases was manual earlier. With latest technologies right from test case generation till result comparison various tools are developed to assist the tester to focus on logical thinking of testing rather than laborious work. Software testing is risk based, because the cost of finding a bug in a later stage of development would be very expensive. Under testing makes the system more prone to errors and over testing makes the system immune to it as said by Beizer, "The more you test a software, the more immune it becomes to your tests". Hence, the responsibility lies on the tester to proactively think and proficiently carry out the testing process to capture as many as defects as possible. In this paper, an overview of software testing trends, techniques and challenges faced by testers are discussed.

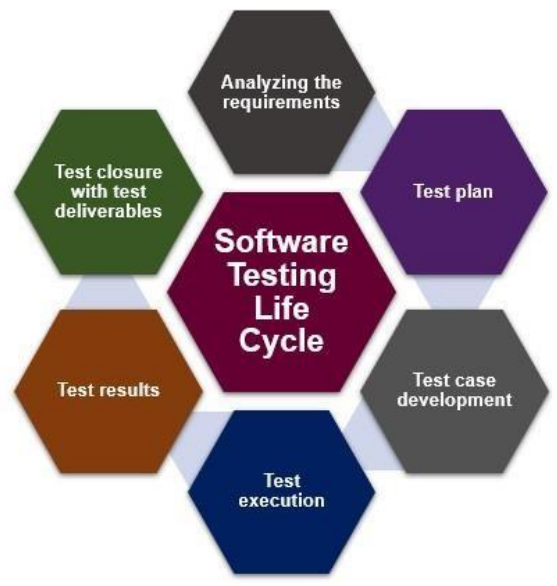

Fig 1. Phases of software testing life cycle

Fig 1. Cycle of software testing which comprises of requirements gathering and analysis, preparing the test plan for the entire test process, writing or scripting test cases, executing the test cases, comparing the results obtained from execution and finally test closure providing all test deliverables.

\section{SOFTWARE TESTING TRENDS, TECHNIQUES, PROBLEMS, AND CHALLENGES}

Software testing trend has evolved rapidly for the past two decades. Software testing process is not an independent entity. It collaborates with development. With various innovations in new methodologies of developing a software or a technology the testing process also marked its transformation. Based on the technology trends, in this paper software testing trends and techniques are covered. (Fig 2. Trends in software testing). 

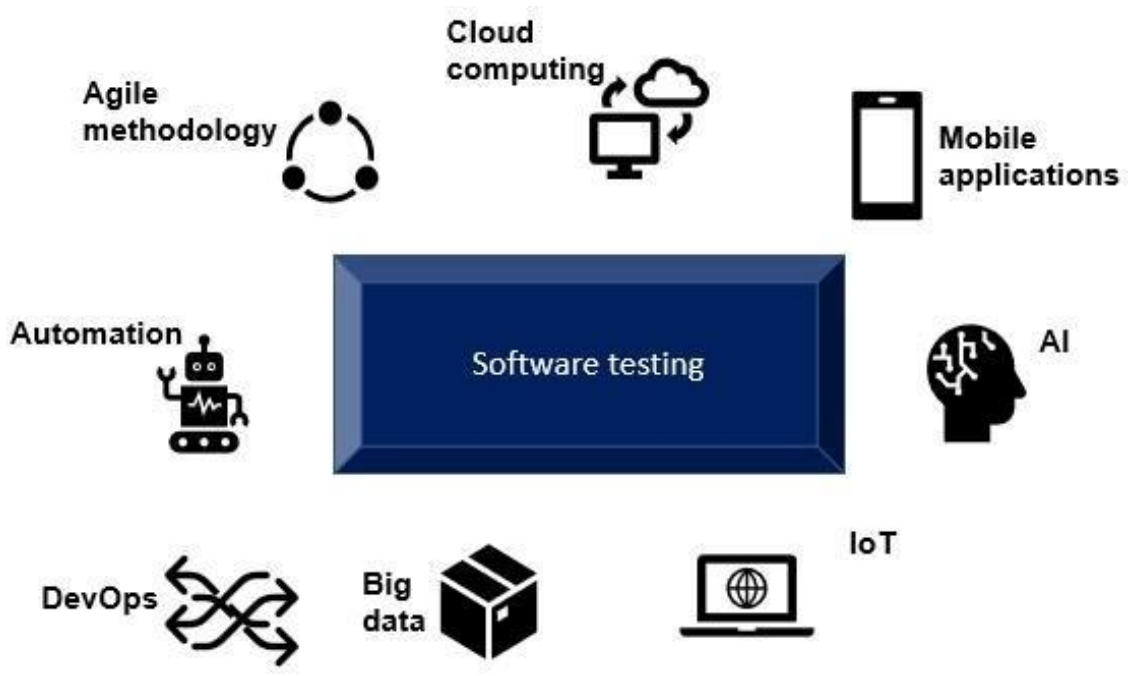

Fig 2. Trends in software testing

Fig 2. Latest technologies like Cloud, AI, IoT, Big Data, DevOps, Automation and Agile changed the way of testing a software. It enhanced the software testing process directing testing to be a though provoking process rather all manual tedious work.

\subsection{Traditional software model}

Waterfall model is a traditional software development model where testing is carried out only after complete development phase. Traditional software testing techniques (TSTT) are used in all trending software methodologies, but the difference lies in when it is used and how it modified and used. Fig 3. Software testing types shows the basic categories of software testing types.

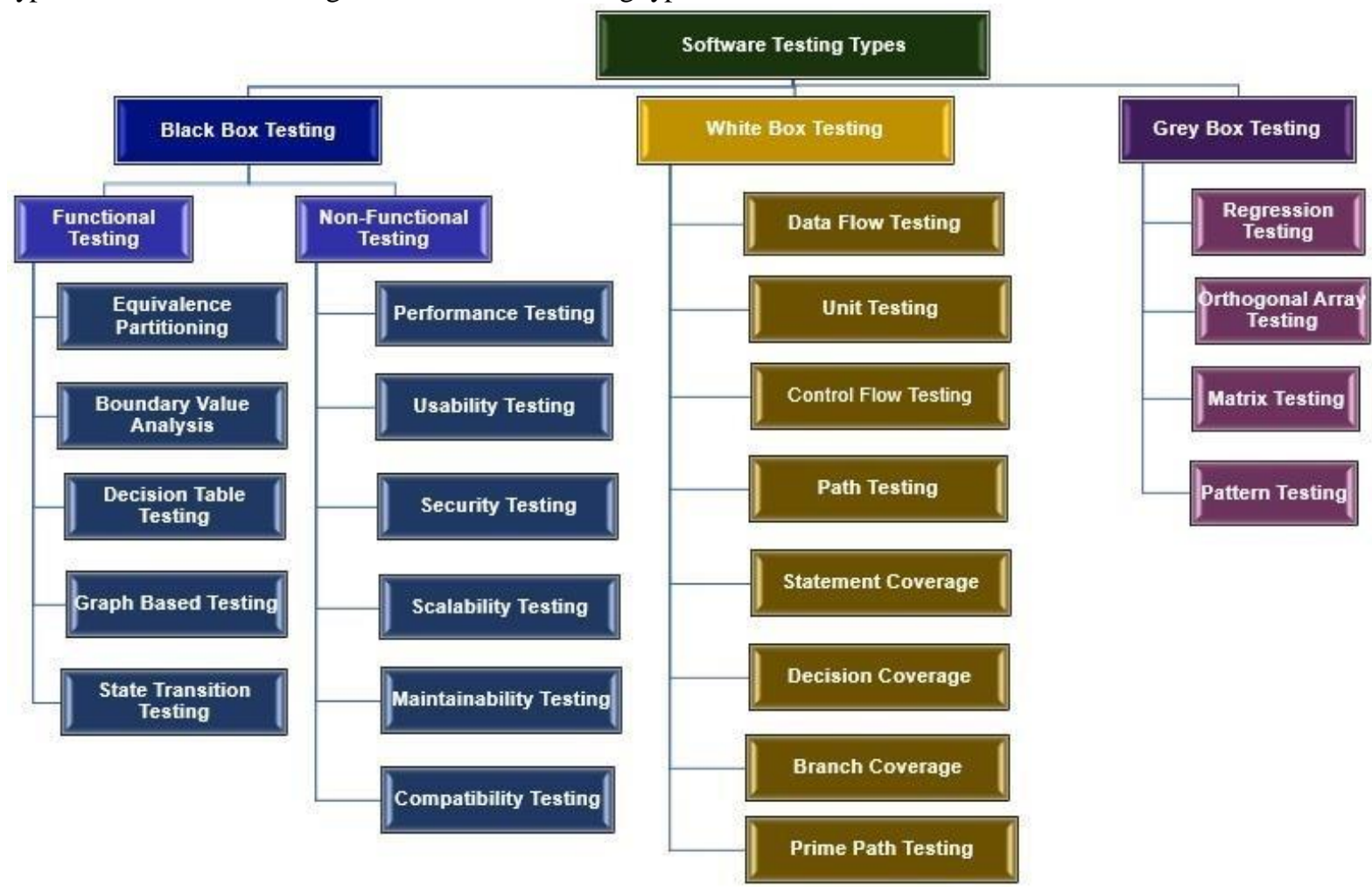

Fig 3. Software testing types 


\section{International Journal of Engineering Applied Sciences and Technology, 2021 \\ Vol. 6, Issue 1, ISSN No. 2455-2143, Pages 94-98 \\ Published Online May 2021 in IJEAST (http://www.ijeast.com)}

Fig 3. Software testing types indicated different types of software testing practiced from traditional waterfall model. If the internal implementation of the system is known and testing is done based on it then it is white box testing. If the tester has no knowledge about the implementation and performs the test, then it is black box testing. Grey box is a combination of both.

\subsection{Automation}

Initially all the software processes were manual, and it is time consuming. The first improvement in software testing was automation where the repeated test execution can be performed without manual intervention and the results would be automatically compared. The testing process is made quite easier now because right from test case generation, test data, test execution are all automated. In automation, TSTT are automated using various tools and other popular automation software testing techniques are test automation pyramid, record/playback, scripting, data-driven testing, keyword-based, API testing, parallel testing, key word testing, socket programming testing, web service testing. With growing largescale systems doing entire testing process automated ( $\mathrm{Li}$ et al,2019), maintaining the entire test set is tedious, time consuming and increases the complexity of the process. Proper selection of testing approach and potential financial risks of false results due to automation are the common challenges.

\subsection{Agile}

Testing began in parallel with the development process in agile software development methodology. This marked another significant trend in software testing as the testing is done right from the beginning of the development process, making it iterative and adaptive unlike traditional testing. Based on the incremental and adaptive nature of agile methodology in addition to TSTT it has acceptance test-driven development, behavior-driven development testing (Perera et al, 2017), exploratory testing, and session-based testing techniques. Regression testing techniques are vital in agile as the system undergoes many short releases. One of the agile main principles in Agile manifesto is welcoming changing requirements. But if there is high frequency of requirement changing then testing would be hefty as regression testing would itself make it time critical. If the requirements are not defined properly then the entire product would fail as it goes through rapid changes making the entire quality process tough and a failure. Also, due to time constraint there is a limited or no security or other non- functional testing. There are various types of regression testing techniques used still testers face the issue of flaky tests, which can both pass and fail when run multiple times on the same version of code and tests. (Lam et al,2020).

\subsection{Mobile applications}

Testing process saw its implementation in mobile applications too where the platforms for testing differ with the basic functionality being same. In addition to TSTT, installation testing, auto tests, interface testing, memory leakage testing, interrupt testing and certification testing techniques are used to test the mobile applications. There are several issues faced in testing mobile applications like OS fragmentation, testing on various screen sizes of mobile, responsiveness, different mobile networks, interfaces, and usability. Though there are simulators for such testing it does not reflect realtime scenario. The scope is limited.

\subsection{Big data technology}

As the systems grow their data grow by increasing the complexity of the data systems that paved the way for big data. Big data was entirely different from the above testing approaches as big data testing requires testing of large volumes of both structured and unstructured data. Automation algorithms help in getting Value for big data. Here the large data sets are tested for their five V's not the system functionally alone. Data analytics, visualisation testing (Volume of data), big data eco system testing (Veracity of data), migration source extraction testing (Velocity of data), performance and security (Variety of data) are big data specific testing techniques. Main challenges of big data testing lie in testing heterogenous and vast data, understanding the data relationship, security, and scalability.

\subsection{Cloud computing}

Cloud computing and big data are inter-related where cloud computing access the data from a remote location and process it (Wang et al,2017). Cloud testing is done by simulating real world data web traffic data to test web applications in cloudbased environment. Disaster recovery testing, multi- tenancy testing, security testing, load balancing, clustering techniques are most specific techniques in testing cloud. Data is collected through various platforms accessed from different servers. While testing is a real challenge to estimate the accuracy and consistency of the data as it has passed through several channels of communication. Data integrity is a big concern. Also, issues arise in complexity of scalability, storage, and migration of data between clouds (Wang et al,2017). 


\section{International Journal of Engineering Applied Sciences and Technology, 2021 \\ Vol. 6, Issue 1, ISSN No. 2455-2143, Pages 94-98 \\ Published Online May 2021 in IJEAST (http://www.ijeast.com)}

\subsection{DevOps and QAOps}

DevOps testing streamlined both agile and automation testing trend making the entire software life cycle automated fusing QAOps framework with it by having Continuous Integration/Continuous Delivery. It includes all agile testing techniques mainly automation testing, regression, integration, parallel testing, exploratory, T-shaped (Faber,2020) and scalability testing techniques. With large codes, it is difficult to cover $100 \%$ code in testing. In technologies like DevOps there is an urge for continuous integration and delivery. Time, a critical factor testing could be really challenging to cover the entire code within such short span of deliverables.

\subsection{Artificial Intelligence (AI)}

Now it is the giant leap in software testing process with the advent of artificial intelligence and machine learning. Right from test case generation, test data collection, execution and result comparison are all managed by heuristic AI algorithms. AI is mutual to software testing as AI improves the efficiency of software testing by using ML algorithms to perform testing as well as software plays a key role in AL systems. Block chain testing, Smart Contract Testing, Peer/Node Testing, Hyper Automation, Cybersecurity mesh testing, rule- based testing, classification testing, data model-based testing, Metamorphic (non-oracle) testing (Gao et al,2019), chat box testing, code less automation testing, cross over and mutation testing in genetic algorithms, fuzz testing, ML testing techniques like supervised, unsupervised and reinforcement testing are commonly used testing techniques.

Software testing now includes testing of dynamically generated reactive test data. Here arises the problem of determining the correct output for the input which is known as Test Oracle problem. This is prevalent in $\mathrm{AI}$ and ML where they are generally non-deterministic where you cannot expect same output for same input. Machine learning algorithms are complex to test as they rely on human inputs, and it relies on volume of training data to get predictions.

Test assessment criteria, setting testing benchmarks and search-based software test generation are other challenges.

\subsection{Internet of Things}

Internet of Things is accelerating in past 3 to 4 years where testing IoT things involves testing IoT device with software, its relevant peripherals and communication between them. This requires specialised testing skillset consisting knowledge of all components of IoT. IoT devices have four basic components namely sensor, application, network, and back end. In addition to above techniques sensor testing, synchronization, interoperability testing, device to device testing, interrupt testing is more specific to IoT testing. With IoT seeing tremendous emergence in recent years, security testing is a major challenge for the software testers. There are many vulnerable points for various devices integrated into the IoT systems as the number of links for connected device points would grow rapidly. Cross device compatibility testing is another concern. Standardized testing techniques are not framed for IoT. Here apart from security, the tester must have deep knowledge in software, hardware, interfaces and specially networks. Needs arise to have quality people with all this deep domain knowledge would be a pressure (Bures et al, 2018)

\section{CONCLUSION}

Market trend is shifting towards interrelated to AI, IoT, agile, cloud, block chain, virtual reality, mobile apps, edge computing, robotics etc., Tester should have strong expertise in programming language to test all trending technologies where coding becomes indispensable in its automation. Software testing is to ensure the quality of the product. Software testing techniques can be view from many dimensions like technology used, stake holders, functionality, development methodologies or the phase of development itself. This paper has covered common testing techniques and challenges in software testing arena. More standard generalised software testing specific models could be developed to overcome the challenges faced in testing fragmented platforms.

\section{REFERENCES}

1. J.Jenny Li, Andreas Ulrich, Xiaoying Bai \& Antonia Bertolino, 2019. Advances in test automation for software with special focus on artificial intelligence and machine learning. Software Quality Journal.

2. Pulasthi Perera, Roshali Silva \& Indika Perera, 2017. Improve Software Quality through Practicing DevOps. International Conference on Advances in ICT for Emerging Regions.

3. Wing Lam,August Shi,Reed Oei,Sai Zhang,Michael D. Ernst \& Tao Xie, 2020. Dependent-Test-Aware Regression Testing Techniques. ISSTA '20, Association for Computing Machinery.

4. Lizhe Wang, Rajiv Ranjan, Jinjun Chen and 
Boualem Benatalla, 2011. Cloud Computing: Methodology, Systems, and Applications. CRC Press, Taylor \& Francis group.

5. Frank Faber, 2020. Testing in DevOps. In: Goericke S. (eds) The Future of Software Quality Assurance.

6. Jerry Gao, Chuanqi Tao, Dou Jie \& Shenqiang, 2019. What is AI Software Testing? and Why? 2019 IEEE International Conference on Service-Oriented System Engineering (SOSE).

7. Miroslav Bures, Tomas Cerny \& Bestoun S. Ahmed, 2018. Internet of Things: Current Challenges in the Quality Assurance and Testing Methods. 9th iCatse Conference on Information Science and Applications 2018. 